\title{
Testosterone therapy in female-to-male transsexuals: effects on body weight, blood pressure and lipid profile
}

Carmen Acosta Calero ${ }^{1}$, Claudia Arnás León ${ }^{1}$, Ana Delia Santana Suárez ${ }^{1}$, Sara Quintana Arroyo ${ }^{1}$, Francisco Javier Martínez Martín²

${ }^{1}$ Endocrinology \& Nutrition Dpt., Hospital Universitario de Gran Canaria Dr. Negrín, Las Palmas de Gran Canaria, Spain; ${ }^{2}$ Endocrinology Dpt., Hospitales San Roque, Las Palmas de Gran Canaria, Spain

\section{Introduction}

-A possible increase in blood pressure (BP) and other cardiovascular risk factors has been associated with testosterone treatment (TT) in female-to-male transsexuals (FMT). Nonetheless, the available evidence does not support an increase in CV morbidity or mortality.

-We set out to analyze the impact of TT on BP and lipid profile in a cohort of FMT patients.

\section{Methodology}

- Retrospective observational study with no control group in a cohort of 34 FMT patients who started TT following a standard protocol, comparing clinical and routine laboratory data before and after 6-12 months.
Results

-Age was 276 years and BMI $26.73 .1 \mathrm{~kg} / \mathrm{m} 2$.

- As expected, treatment increased free testosterone levels and reduced 17- $\beta$ estradiol. BMI increased to $27.83 .4 \mathrm{~kg} / \mathrm{m}^{2}$ ( $\mathrm{p}$ $<0.001)$

- There were no significant changes in systolic BP (120.9 9.9 to $120.610 .9 \mathrm{mmHg})$ and diastolic BP (82.4 7.6 to $80.98 .1 \mathrm{mmHg}$ ), glycemia, creatinine and total cholesterol (TC), but HDL-cholesterol was significantly reduced (58.9 12.9 to $54.713 .6 \mathrm{mg} / \mathrm{dL}, \quad \mathrm{p}<0.001)$ while LDL-cholesterol (99.9 23.9 to $103.523 .0 \mathrm{mg} / \mathrm{dL}, \mathrm{p}=0.030$ ) and triglycerides (166.6 37.8 to $178.145 .2 \mathrm{mg} / \mathrm{dL}, \mathrm{p}<0.001$ ) were increased.

- There were no new-onset hypertension cases.

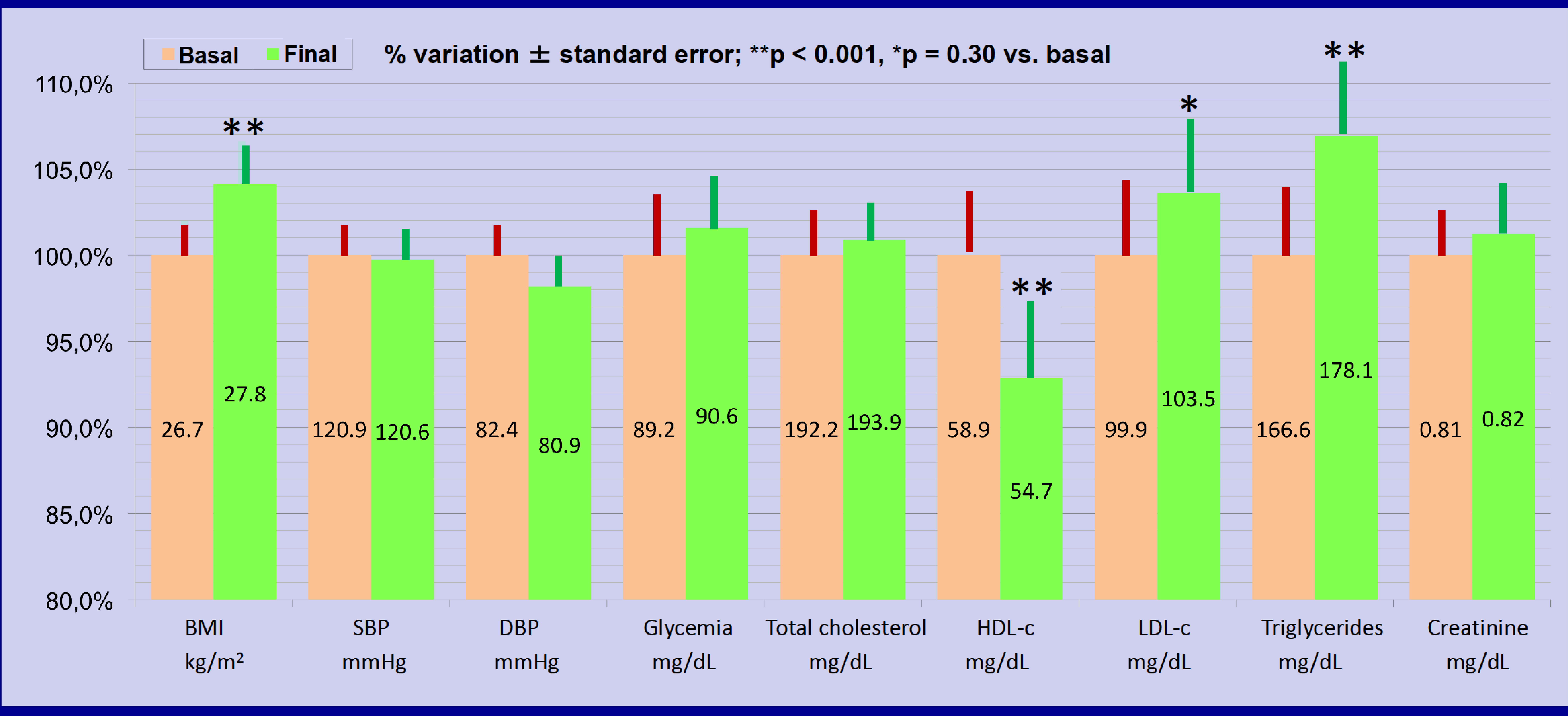

Conclusions

- Our study does not support the BP increase reported in some series. We found, however an increased BMI. In the literature, TT is associated with increased muscle mass but not adiposity, thus we cannot infer an increased cardiovascular risk. Regrettably we do not have impedanciometry data to elucidate this point.

- There is a mild but definite deterioration of the lipid profile with reduced HDL-cholesterol and increased LDL-cholesterol and triglycerides. That may be in part attributed to reduced levels of 17- $\beta$-estradiol rather than a direct action of testosterone on lipid metabolism. This is however considered as a common adverse effect of TT. 